The Annals of Applied Probability

2002, Vol. 12, No. 1, 69-100

\title{
TAIL PROBABILITIES OF SUBADDITIVE FUNCTIONALS OF LÉVY PROCESSES ${ }^{1}$
}

\author{
By Michael BraVerman, ${ }^{2}$ ThOMAS MiKosCH ${ }^{3}$ \\ AND GENNADY SAMORODNITSKY ${ }^{4}$ \\ Ben-Gurion University of the Negev, University of Copenhagen \\ and Cornell University
}

\begin{abstract}
We study the tail behavior of the distribution of certain subadditive functionals acting on the sample paths of Lévy processes. The functionals we consider have, roughly speaking, the following property: only the points of the process that lie above a certain curve contribute to the value of the functional. Our assumptions will make sure that the process ends up eventually below the curve. Our results apply to ruin probabilities, distributions of sojourn times over curves, last hitting times and other functionals.
\end{abstract}

1. Introduction. Both in the theory and in applications of stochastic processes one is often interested in two types of questions: When does the process $\mathbf{X}=$ $\{X(t), t \geq 0\}$ lie above a certain deterministic function (curve) $\boldsymbol{\mu}=\{\mu(t), t \geq 0\}$, and given the process exceeds this curve, what are its values? For example, what can be said about the distribution of the biggest excess of the process over the curve and, if both the process and the function are measurable, what is the distribution of the time the process spends above the curve?

In this paper, we outline a general approach to the asymptotic tail behavior of the distributions of these and other subadditive functionals acting on an infinitely divisible process with "not too light" tails. (The latter notion will be made precise soon.) We focus on a particular class of infinitely divisible processes, the well-known Lévy processes, and we consider the distributional tails of various subadditive functionals of their paths. These examples will show in detail how successfully this method works and how general it is.

Received April 2000; revised March 2001.

${ }^{1}$ Research supported by NATO Collaborative Research Grant CRG 971579 and by EURANDOM.

${ }^{2}$ Research supported in part by a Gilady fellowship.

${ }^{3}$ Research supported by DYNSTOCH and SNF Research Grant 21-01-0546.

${ }^{4}$ Research supported in part by NSF Grants DMS-97-04982 and DMI-97-13549 and by NSA Grant MDA904-98-1-0041 at Cornell University.

AMS 2000 subject classifications. Primary 60E07; secondary 60K30, 60G10.

Key words and phrases. Ruin probability, heavy tails, supremum, negative drift, risk, subadditive functional, sojourn time, last hitting time, Lévy process. 
Let $\mathbf{X}$ be an infinitely divisible process without Gaussian component and Lévy measure $v$. Following Maruyama (1970), the distribution of $\mathbf{X}$ is characterized as follows:

$$
E e^{i\langle\boldsymbol{\beta}, \mathbf{X}\rangle}=\exp \left\{\int_{\mathbb{R}^{[0, \infty)}}\left(e^{i\langle\boldsymbol{\beta}, \boldsymbol{\alpha}\rangle}-1-i\langle\boldsymbol{\beta}, \boldsymbol{\tau}(\boldsymbol{\alpha})\rangle\right) v(d \boldsymbol{\alpha})+i\langle\boldsymbol{\beta}, \boldsymbol{\gamma}\rangle\right\}
$$

$$
\boldsymbol{\beta} \in \mathbb{R}^{([0, \infty))} .
$$

Here $v$ is the projective limit of the Lévy measures corresponding to the finite dimensional distributions of $\mathbf{X}$ and $\boldsymbol{\gamma} \in \mathbb{R}^{[0, \infty)}$. The symbol $\mathbb{R}^{([0, \infty))}$ denotes the space of real functions $\boldsymbol{\beta}$ defined on $[0, \infty)$ such that $\beta(t)=0$ for all but finitely many $t$, and $\langle\boldsymbol{\beta}, \boldsymbol{\alpha}\rangle=\sum_{t \in[0, \infty)} \beta(t) \alpha(t)$. Finally, $\tau(\boldsymbol{\alpha})(t)=\alpha(t) \mathbb{1}(|\alpha(t)| \leq 1)$.

Some examples of the measurable functionals $\phi: \mathbb{R}^{[0, \infty)} \rightarrow(-\infty, \infty]$ on $\mathbf{X}$ we consider are

$$
\begin{aligned}
\phi_{\text {sup }}(\boldsymbol{\alpha}) & =\sup _{t \geq 0}[\alpha(t)]_{+}, \quad \phi(\boldsymbol{\alpha})=\sup \{t>0: \alpha(t)>0\}, \\
\phi(\boldsymbol{\alpha}) & =\int_{0}^{\infty}[\alpha(s)]_{+}^{p} d s,
\end{aligned}
$$

where $y_{+}=\max (0, y)$ and $p \in(0,1]$. The supremum functional $\phi_{\text {sup }}$ has gained particular importance in the context of queuing and insurance, where one is interested in quantitative measures for the excesses of $\mathbf{X}$ over high level thresholds, which event is interpreted as buffer overflow or ruin in the different contexts. The above functionals have in common that they are subadditive, that is, for any $\boldsymbol{\alpha}_{1}, \boldsymbol{\alpha}_{2} \in \mathbb{R}^{[0, \infty)}$,

$$
\phi\left(\boldsymbol{\alpha}_{1}+\boldsymbol{\alpha}_{2}\right) \leq \phi\left(\boldsymbol{\alpha}_{1}\right)+\phi\left(\boldsymbol{\alpha}_{2}\right) .
$$

If, with probability $1, \phi(\mathbf{X}-\boldsymbol{\mu})<\infty$ is finite, it makes sense to measure the thickness of the distributional tail $P(\phi(\mathbf{X}-\boldsymbol{\mu})>u)$ for large $u$. Suppose this tail does not decay "too fast" as $u \rightarrow \infty$ and define

$$
\psi(u)=v(\{\boldsymbol{\alpha}: \phi(\boldsymbol{\alpha}-\boldsymbol{\mu})>u\}) .
$$

The subadditivity of the functional $\phi$, the presence of heavy tails and the logic of large deviations, saying that unlikely events happen in the most likely way, lead one to the conjecture that $\psi(u)$ and $P(\phi(\mathbf{X}-\boldsymbol{\mu})>u)$ are equivalent in the following sense:

$$
\lim _{u \rightarrow \infty} \frac{P(\phi(\mathbf{X}-\boldsymbol{\mu})>u)}{\psi(u)}=1 .
$$

Indeed, relations of type (1.4) were proved in the theory of laws with so-called subexponential tails. For example, Embrechts, Goldie and Veraverbeke (1979) 
considered the overall supremum of Lévy processes, and Rosiński and Samorodnitsky (1993) studied very general subadditive functionals.

The setup in the latter paper is, in fact, close to the present one. However, there is one crucial difference: the functionals in Rosiński and Samorodnitsky (1993) were assumed to be bounded by an almost surely finite pseudonorm of the process. Hence these processes are, in a certain sense, bounded "from above and below." This assumption is far away from the situation in the present paper. Our functionals are akin to the supremum of a negative drift random walk over the entire infinite horizon. In this sense, they are bounded "only from one side."

The validity of relation (1.4) has been established for the overall supremum functional $\phi_{\text {sup }}$ and some particular classes of processes with subexponential tails. Those include Lévy processes with a negative linear drift [see Embrechts and Veraverbeke (1982)] and symmetric $\alpha$-stable processes, $\alpha \in(1,2)$, with stationary ergodic increments and negative linear drift. In general, the precise circumstances under which (1.4) is valid for subadditive functionals are not known, even in the particular case of Lévy processes. The results of the present paper provide a further step in the process of understanding the tail equivalence relation (1.4) for heavy tailed processes. Once again, we will focus on Lévy processes and a large family of subadditive functionals $\phi$ and deterministic functions $\boldsymbol{\mu}$.

The proof of our main result (Theorem 3.1) shows that we use the "heavy tail large deviations heuristics." This means that large values of the functional $\phi(\mathbf{X}-\boldsymbol{\mu})$ are essentially due to one very large jump of the Lévy process that occurs early enough, before the negative drift took it "too far down." Since Lévy processes are well described by Poisson processes, we make extensive use of the latter tool. In particular, we show that the large deviation idea can be made precise by considering the "large and occurring early enough" jumps and the "small or occurring too late" jumps of the underlying Poisson process separately. This leads one to a decomposition of the Lévy process into two independent processes. We show that the process which represents the small jumps is asymptotically negligible; that is, this process will not contribute to the asymptotic tail behavior of $\phi(\mathbf{X}-\boldsymbol{\mu})$. The crucial part in this decomposition is the process which represents the large jumps of the Lévy process. It has representation as a compound Poisson sum of paths. We show that the tail behavior of $\phi(\mathbf{X}-\boldsymbol{\mu})$ is essentially determined by a single term in that sum.

In related work Hüsler and Piterbarg (1999) considered the tail behavior of the supremum functional $\phi_{\text {sup }}$ of certain Gaussian processes, including fractional Brownian motion, with negative (not necessarily linear) drift. The Gaussian nature of the underlying process causes exponential decay of the tails $P\left(\phi_{\text {sup }}(\mathbf{X}-\boldsymbol{\mu})>u\right)$.

This paper is organized as follows. In Section 2 we give a reasonably transparent set of assumptions we impose on the family of the subadditive functionals $\phi$, the function (curve) $\boldsymbol{\mu}$ and the distribution of the Lévy processes $\mathbf{X}$. There we also start looking at some basic examples of subadditive functionals which were the 
motivation for our research. The conditions include regular variation of $\boldsymbol{\mu}$ and of the tails of the Lévy measure of $\mathbf{X}$. However, our main theorem holds under assumptions more general than regular variation and can be substantially relaxed. For the ease of presentation, a set of such conditions is given in Section 4, where we also relate the parameters of $\boldsymbol{\mu}, \mathbf{X}$ and $\phi$. The most general conditions under which we could prove the main theorem are stated in Section 7. The main result on the asymptotic behavior of the tails $P(\phi(\mathbf{X}-\boldsymbol{\mu})>u)$ is formulated in Section 3. It describes one situation when relation (1.4) is valid. In this section we also continue the discussion of examples which we started in Section 2 and give additional examples. We explicitly calculate the tail asymptotics for a number of important subadditive functionals acting on Lévy processes. Among those are the functionals in (1.2), but also, for example, the sojourn time of a Lévy process above a curve. The main steps of the proof are given in Section 5. However, the proof is quite technical and therefore we postpone various calculations until Section 6.

2. Assumptions and notation. Throughout this paper, $C$ stands for a generic positive constant $C$. Its value will be allowed to change from appearance to appearance, even if we do not mention it explicitly.

Let $\mathbf{X}=\{X(t), t \geq 0\}$ be a Lévy process, that is, a real-valued process with stationary and independent increments, and Lévy measure $\rho$ on $\mathbb{R}$. We refer the reader to Bertoin (1996) and Sato (1999) for encyclopedic treatments of Lévy processes. In particular, one can find detailed proofs of the properties we mention and use below.

Specifically, the marginal distributions of a Lévy process are determined by the characteristic function which, in turn, is determined by its one-dimensional Lévy measure and drift. If the Lévy process has finite means, we will assume the mean to be zero, and so write the characteristic function in the form

$$
E e^{i \theta X(1)}=\exp \left\{\int_{-\infty}^{\infty}\left(e^{i \theta x}-1-i \theta x\right) \rho(d x)\right\}, \quad \theta \in \mathbb{R},
$$

whereas in the case of infinite mean, the drift turns out to be unimportant for our applications, and we will assume that the characteristic function of the process has the form

$$
E e^{i \theta X(1)}=\exp \left\{\int_{-\infty}^{\infty}\left(e^{i \theta x}-1-i \theta x \mathbb{1}(|x| \leq 1)\right) \rho(d x)\right\}, \quad \theta \in \mathbb{R} .
$$

We always take a version of $\mathbf{X}$ with all sample paths in the Skorohod space $\mathbb{D}[0, \infty)$, that is, with paths which are right-continuous at every $t \geq 0$ and have left limits at every $t>0$. This version of $\mathbf{X}$ is automatically measurable; this feature will become useful because we will have many opportunities to integrate the sample paths of $\mathbf{X}$. 
If one writes the characteristic function of a Lévy process process $\mathbf{X}$ in the form (1.1), then the corresponding Lévy measure $v$ has the form

$$
v(A)=\int_{0}^{\infty} \int_{-\infty}^{\infty} \mathbb{1}\left(x \mathbb{1}_{[s, \infty)} \in A\right) \rho(d x) d s
$$

for any measurable set $A \subset \mathbb{R}^{[0, \infty)}$. Therefore the function $\psi$ in (1.3) turns into

$$
\psi(u)=\int_{0}^{\infty} \int_{-\infty}^{\infty} \mathbb{1}\left(\phi\left(x \mathbb{1}_{[s, \infty)}-\boldsymbol{\mu}\right)>u\right) \rho(d x) d s, \quad u>0 .
$$

We denote the right tail of the one-dimensional Lévy measure $\rho$ by

$$
H(u)=\rho([u, \infty)), \quad u>0 .
$$

Since we aim at readability, we will not assume the most general sufficient conditions under which our results hold. However, these assumptions can be substantially relaxed. A set of such conditions can be found in Section 7 and the corresponding proofs are available in an extended version of the present paper in Braverman, Mikosch and Samorodnitsky (2000).

2.1. Assumptions on the Lévy measure $\rho$. We assume that the following hold:

$$
H \text { is regularly varying with exponent }-\alpha \text { for some } \alpha>0 \text {; }
$$

there is a constant $C>0$ such that

$$
\rho((-\infty,-t]) \leq C \rho([t, \infty)) \text { for all } t \geq 1 .
$$

2.2. Assumptions on the drift $\boldsymbol{\mu}$. We assume that

(2.7) $\mu$ is regularly varying with exponent $\beta$ for some $\beta>\max \left(\alpha^{-1}, 0.5\right)$.

2.3. Assumptions on the subadditive functional $\phi$. Let $\phi: \mathbb{R}^{[0, \infty)} \rightarrow[0, \infty]$ be a measurable subadditive functional satisfying the following conditions:

1. The functional "lives off only positive values of its argument"-This means that

$$
\phi(\mathbf{0})=0, \quad \text { and if } \alpha(t) \leq 0 \text { for all } t>t_{0}, \text { some } t_{0} \text {, then }
$$

$$
\phi(\boldsymbol{\alpha})=\phi\left(\boldsymbol{\alpha} \mathbb{1}_{\left[0, t_{0}\right]}\right) .
$$

Here $\boldsymbol{\alpha} \mathbb{1}_{\left[0, t_{0}\right]}=\left\{\alpha(t) \mathbb{1}_{\left[0, t_{0}\right]}(t), t \geq 0\right\}$.

2. The functional is finite on locally bounded functions that are eventually nonpositive-This means that

$$
\begin{array}{cl}
\phi(\boldsymbol{\alpha})=\phi\left(\boldsymbol{\alpha} \mathbb{1}_{\left[0, t_{0}\right]}\right)<\infty & \text { if } \alpha(t) \leq 0 \text { for all } t>t_{0}, \text { some } t_{0}, \\
& \text { and } \sup _{t \leq t_{0}} \alpha(t)<\infty .
\end{array}
$$


3. Monotonicity-This means that

$$
\text { if } \alpha(t) \leq \beta(t) \text { for all } t \text {, then } \phi(\boldsymbol{\alpha}) \leq \phi(\boldsymbol{\beta})
$$

and

$$
\phi(c \boldsymbol{\alpha}) \leq \phi(\boldsymbol{\alpha}) \quad \text { for all } c \in[0,1] \text { and } \boldsymbol{\alpha} \in \mathbb{R}^{[0, \infty)} .
$$

Notice that (2.11) is implied by (2.10) if $\alpha(t) \geq 0$ for all $t \geq 0$.

The reader who wants to learn about some relevant examples of subadditive functionals $\phi$ satisfying the above conditions is referred to Section 3.

2.4. Function $T(s, u)$. Crucial information describing the behavior of the tail probability $P(\phi(\mathbf{X}-\boldsymbol{\mu})>u)$ is contained in the following function. For $s \geq 0$ and $u>0$ define

$$
T(s, u)=\inf \left\{x>0: \phi\left(x \mathbb{1}_{[s, \infty)}-\boldsymbol{\mu}\right)>u\right\},
$$

and denote

$$
T(u)=T(0, u) .
$$

To get some feeling about the behavior of these functions, let us consider several examples. In these examples we assume, for simplicity, that the drift function $\boldsymbol{\mu}$ is monotone. Our main theorem below is applicable to these examples irrespective of $\boldsymbol{\mu}$ being monotone. See the discussion below.

EXAMPLE 2.1. One of the interesting subadditive functionals is the overall supremum

$$
\phi_{\text {sup }}(\boldsymbol{\alpha})=\sup _{t \geq 0} \alpha(t)
$$

It has numerous applications, among which are use in insurance mathematics for describing eventual ruin [see Embrechts, Klüppelberg and Mikosch (1997)] and in queuing for the buffer overflow [see Prabhu (1998)]. It is clear that $\phi_{\text {sup }}$ is a subadditive functional, and that in this case

$$
T(s, u)=\mu(s)+u .
$$

EXAMPLE 2.2. Here one considers the time the process spends above zero:

$$
\phi_{\text {sojourn }}(\boldsymbol{\alpha})=\int_{0}^{\infty} \mathbb{1}(\alpha(t)>0) d t .
$$

It is, once again, clear that $\phi_{\text {sojourn }}$ is a subadditive functional. Furthermore, in this case

$$
T(s, u)=\mu_{0}(s+u),
$$

where $\mu_{0}$ is a certain nondecreasing regularly varying function equivalent to $\boldsymbol{\mu}$; see Lemma 3.2(ii) below. 
EXAMPLE 2.3. Here we consider the last hitting time of zero:

$$
\phi_{\text {last }}(\boldsymbol{\alpha})=\sup \{t>0: \alpha(t) \geq 0\} .
$$

It is not difficult to see that this functional is subadditive and that, in this case,

$$
T(s, u)=\max \left(\mu(s), \mu_{0}(u)\right),
$$

with $\mu_{0}$ as above.

In the examples above one can compute the function $T(s, u)$ explicitly, and our main result, Theorem 3.1, applies to these examples. It turns out, however, that one does not really need to be able to compute the function $T(s, u)$ explicitly, and in some of the further examples below such explicit computation is awkward, or impossible. It is enough to check a few properties of this function; see (4.1)-(4.4) below.

3. The main results. Here we give our main result which was announced in Section 1. The main steps of its proof are given in Section 5. Since the proof is quite technical, we collect some auxiliary results in Section 6.

First recall the definition of the quantity $\psi(u)$ from (2.4).

THEOREM 3.1. Let $\mathbf{X}$ be a Lévy process, $\boldsymbol{\mu}$ a deterministic drift function and $\phi$ a subadditive measurable functional satisfying the following conditions:

(i) The assumptions on the Lévy measure, $\boldsymbol{\mu}$ and $\phi$ in Section 2 hold.

(ii) The function $T(s, u)$ either is one of those given in (2.13)-(2.15) or, more generally, satisfies (4.1)-(4.4) below.

(iii) For $\alpha>1$ and $0<\alpha \leq 1$ in (2.5) the characteristic function of the process is given by (2.1) and (2.2), respectively.

(iv) $\psi(u)$ is regularly varying (at infinity) with exponent $-\alpha<0$.

Then $\psi(u)$ and $P(\phi(\mathbf{X}-\boldsymbol{\mu})>u)$ are equivalent:

$$
\lim _{u \rightarrow \infty} \frac{P(\phi(\mathbf{X}-\boldsymbol{\mu})>u)}{\psi(u)}=1 .
$$

We now apply Theorem 3.1 to compute the asymptotic behavior of the tail probabilities $P(\phi(\mathbf{X}-\boldsymbol{\mu})>u)$ for the examples introduced above, as well as some additional examples.

For easy reference we first collect several well-known facts on regularly varying functions which will be used frequently below. The reader is referred to Bingham, Goldie and Teugels (1987) for proofs and more information. Let

$$
\mu^{\leftarrow}(u)=\sup \{t>0: \mu(t) \leq u\}, \quad u>0,
$$

be the generalized inverse of $\mu$. 
LEMMA 3.2. (i) Let $\boldsymbol{\mu}$ be regularly varying at infinity with positive exponent of regular variation. Then there are monotone functions $\mu_{*}$ and $\mu^{*}$ such that

$$
\mu_{*}(t) \leq \mu(t) \leq \mu^{*}(t) \quad \text { for all } t \geq 0 \quad \text { and } \quad \lim _{t \rightarrow \infty} \frac{\mu^{*}(t)}{\mu_{*}(t)}=1 .
$$

(ii) Let $\boldsymbol{\mu}$ and $\eta$ be regularly varying with positive exponent $\alpha$ of regular variation and such that $\lim _{t \rightarrow \infty} \mu(t) / \eta(t)=1$ (i.e., $\boldsymbol{\mu}$ and $\eta$ are asymptotically equivalent). Then their generalized inverses are regularly varying with exponent $1 / \alpha$ and asymptotically equivalent as well. Moreover, let

$$
\mu_{0}(x)=\inf \left\{y>0: \mu^{\leftarrow}(y)>x\right\}, \quad x>0 .
$$

Then

$$
\lim _{x \rightarrow \infty} \frac{\mu_{0}(x)}{\mu(x)}=1 .
$$

(iii) (Potter's bounds) Let $\boldsymbol{\mu}$ be regularly varying with a positive exponent $\alpha$ of regular variation. For every $C>1$ and $\varepsilon>0$ there is $s_{0}=s_{0}(C, \varepsilon)$ such that, for all $s, t \geq s_{0}$,

$$
\frac{\mu(t)}{\mu(s)} \leq C \max \left(\left(\frac{t}{s}\right)^{\alpha+\varepsilon},\left(\frac{t}{s}\right)^{\alpha-\varepsilon}\right)
$$

3.1. The overall supremum. Here we consider the overall supremum functional of Example 2.1.

THEOREM 3.3. Assume (2.5)-(2.7). Then

$$
\begin{aligned}
P\left(\phi_{\text {sup }}(\mathbf{X}-\boldsymbol{\mu})>u\right) & =P\left(\sup _{t \geq 0}(X(t)-\mu(t))>u\right) \\
& \sim \int_{0}^{\infty} H(\mu(s)+u) d s \sim C(\alpha, \beta) \mu^{\leftarrow}(u) H(u)
\end{aligned}
$$

as $u \rightarrow \infty$. Here $C(\alpha, \beta)=\alpha \int_{0}^{\infty} z^{1 / \beta}(1+z)^{-(1+\alpha)} d z$.

REMARK 3.4. In this paper we deal with "powerlike" tails and, hence, the theorem above is stated under the assumptions of regular variation. We conjecture, however, that the first asymptotic equivalence in (3.6) holds in greater generality, perhaps under the assumption of subexponentiality of the tail of $H$. In fact, if $\alpha>1$ and $\mu(t)=\mu t$, for some $\mu>0$, is a linear function, then the first asymptotic equivalence in (3.6) is just the classical result for the ruin probability as proved by Embrechts and Veraverbeke (1982):

$$
P\left(\sup _{t \geq 0}(X(t)-\mu(t))>u\right) \sim \frac{1}{\mu} \int_{u}^{\infty} H(s) d s,
$$

and the latter result is known to hold when $H$ has a subexponential right tail. 
In fact, it is quite possible that the curve $\mu$ may be allowed to belong to a wider class of functions as well.

Proof of TheOrem 3.3. The first step is to note that it is enough to prove the theorem in the case when $\mu$ is a monotone function. Since all the terms in (3.6) are, obviously, monotone in $\boldsymbol{\mu}$, parts (i) and (ii) of Lemma 3.2 show that, knowing that the theorem holds for monotone functions, implies its validity in general.

Assume, therefore, that $\boldsymbol{\mu}$ is a monotone function. Note that in our case it follows from (2.13), (4.5) and regular variation of $H$ that

$$
\left.\psi(u) \sim \int_{0}^{\infty} H(\mu(s)+u)\right) d s
$$

as $u \rightarrow \infty$, which together with Theorem 3.1 establishes the first asymptotic equivalence in (3.6). Furthermore, by Potter's bounds [Lemma 3.2(iii)], we see, further, that

$$
\left.\psi(u) \sim u^{\alpha} H(u) \int_{0}^{\infty}(\mu(s)+u)\right)^{-\alpha} d s=\alpha u^{\alpha} H(u) \int_{0}^{\infty} \mu^{\leftarrow}(u)(y+u)^{-(\alpha+1)} d y .
$$

Since $\mu \leftarrow$ is, according to Lemma 3.2(ii), regularly varying, the second asymptotic equivalence in (3.6) is a standard exercise in integration of regularly varying functions.

It turns out that the assumptions (4.1), (4.3) and (4.4) are satisfied in this case. Indeed, (4.1) is trivially true, while for every $0<\delta<1$,

$$
\begin{aligned}
\int_{0}^{\infty} H(\delta T(s, \delta u)) d s & =\int_{0}^{\infty} H(\delta(\mu(s)+\delta u)) d s \\
& \leq \int_{0}^{\infty} H\left(\delta^{2}(\mu(s)+u)\right) d s:=I_{\delta}(u) .
\end{aligned}
$$

Since we have already proved that $I_{1}(u)$ is regularly varying, by Potter's bounds, there is a $\theta>0$ such that $I_{1}(u) \geq C u^{-\theta}$ for all $u \geq 1$, while for any $0<\delta<1$ and $u \geq \delta^{-2}$,

$$
I_{\delta}(u) \leq C \delta^{-\theta} I_{1}(u)
$$

In the case $1 \leq u<\delta^{-2}$ we write

$$
I_{\delta}(u)=\int_{\mu(s) \leq \delta^{-2}}+\int_{\mu(s)>\delta^{-2}}:=I_{\delta}^{(1)}(u)+I_{\delta}^{(2)}(u) .
$$

Using Potter's bounds in the same way as before shows that

$$
I_{\delta}^{(1)}(u) \leq C \delta^{-\theta} I_{1}(u) .
$$


Moreover, since $H$ is the tail of a Lévy measure, we know that, for some $C>0$, $H(y) \leq C y^{-2}$ for all $0<y \leq 2$. Therefore, by the regular variation of $\boldsymbol{\mu}$,

$$
I_{\delta}^{(1)}(u) \leq C \delta^{-4} u^{-2} \int_{0}^{\infty} \mathbb{1}\left(\mu(s) \leq \delta^{-2}\right) d s \leq C \delta^{-\theta_{1}}
$$

for some $\theta_{1}>0$. Putting everything together establishes that, for some $C>0$ and $\theta_{2}>0$,

$$
\int_{0}^{\infty} H(\delta T(s, \delta u)) d s \leq C \delta^{-\theta_{2}} \int_{0}^{\infty} H(T(s, u)) d s
$$

for all $u \geq 1$ and $0<\delta<1$. This is, of course, more than enough to prove (4.4). Finally, the assumption (4.3) is an immediate consequence of (3.7) and Potter's bounds.

3.2. The time the process spends above zero. In this section we consider the sojourn time above zero of Example 2.2.

THEOREM 3.5. Assume (2.5)-(2.7). Then

$$
P\left(\phi_{\text {sojourn }}(\mathbf{X}-\boldsymbol{\mu})>u\right)=P\left(\int_{0}^{\infty} \mathbb{1}(X(t)-\mu(t)>0) d t>u\right)
$$

$$
\sim \int_{u}^{\infty} H(\mu(s)) d s \sim C(\alpha, \beta) u H(\mu(u))
$$

as $u \rightarrow \infty$. Here $C(\alpha, \beta)=(\alpha \beta-1)^{-1}$.

Proof. We may and will assume that $\boldsymbol{\mu}$ is monotone. Furthermore, we may assume that $\mu(0) \geq 1$. Indeed, let

$$
\tilde{\mu}(t)=\max (\mu(t), 1+\log (1+t)), \quad t \geq 0 .
$$

Then $\tilde{\mu}(0) \geq 1, \tilde{\mu}(t) \sim \mu(t)$ as $t \rightarrow \infty$, and it is easy to check that monotonicity and subadditivity of the functional $\phi_{\text {sojourn }}$ imply that

$$
P\left(\phi_{\text {sojourn }}(\mathbf{X}-\boldsymbol{\mu})>u\right) \sim P\left(\phi_{\text {sojourn }}(\mathbf{X}-\tilde{\boldsymbol{\mu}})>u\right)
$$

as $u \rightarrow \infty$.

Note that in this case $T(s, u)$ is given by (2.14) [with $\mu_{0}$ as in Lemma 3.2(ii)], and so it follows from that part of the lemma, (4.5) and regular variation of $H$ that

$$
\psi(u) \sim \int_{u}^{\infty} H(\mu(s)) d s
$$

as $u \rightarrow \infty$. 
Once again, the conditions (4.1), (4.3) and (4.4) hold in this case. Indeed, (4.1) follows from Proposition 4.2. Furthermore, we have

$$
\int_{0}^{\infty} H(\delta T(s, \delta u)) d s=\int_{\delta u}^{\infty} H\left(\delta \mu_{0}(s)\right) d s=\delta \int_{u}^{\infty} H\left(\delta \mu_{0}(\delta s)\right) d s:=I_{\delta}(u) .
$$

Note that the assumption $\mu(0) \geq 1$ implies that $\mu_{0}(0) \geq 1$. Therefore, if $0<\delta<1$ and $u \geq 1 / \delta$ we can use Potter's bounds and the fact that $H(y) \leq C y^{-2}$ for all $0<y \leq 2$ to see that for some $C>0$ and $\theta>0$ we have

$$
I_{\delta}(u) \leq C \delta^{-\theta} I_{1}(u)
$$

whereas if $1 \leq u<1 / \delta$, then

$$
I_{\delta}(u) \leq C \delta^{-\theta}\left(1+I_{1}(u)\right) .
$$

The already established regular variation at infinity of $I_{1}(u)$ implies now that

$$
I_{\delta}(u) \leq C \delta^{-\theta} I_{1}(u)
$$

for all $0<\delta<1$ and $u \geq 1$, which is, once again, more than enough to prove (4.4). The assumption (4.3) is an immediate consequence of Potter's bounds.

3.3. The last hitting time of zero. In this section we consider the last hitting time functional of Example 2.3.

THEOREM 3.6. Assume (2.5)-(2.7). Then

$$
P\left(\phi_{\text {last }}(\mathbf{X}-\boldsymbol{\mu})>u\right)=P(\sup \{t>0: X(t) \geq \mu(t)\}>u)
$$

$$
\sim u H(\mu(u))+\int_{u}^{\infty} H(\mu(s)) d s \sim C(\alpha, \beta) u H(\mu(u))
$$

as $u \rightarrow \infty$. Here $C(\alpha, \beta)=1+(\alpha \beta-1)^{-1}$.

Proof. The proof is similar to the one for Theorem 3.5. Without loss of generality we may and will assume that $\boldsymbol{\mu}$ is monotone increasing and $\mu(0)$, $\mu_{0}(0) \geq 1$.

Notice that

$$
\phi_{\text {last }}\left(x \mathbb{1}_{[s, \infty)}-\boldsymbol{\mu}\right)= \begin{cases}0, & \text { if } x \leq \mu(s) \\ \mu^{\leftarrow}(x), & \text { if } x>\mu(s)\end{cases}
$$

Therefore $T(s, u)$ is given by (2.15), and so we may conclude that

$$
\psi(u) \sim u H\left(\mu_{0}(u)\right)+\int_{u}^{\infty} H(\mu(s)) d s,
$$

which together with Karamata's theorem concludes the proof of the theorem. 
In this case the conditions (4.1), (4.3) and (4.4) hold as well, with (4.1) following from Proposition 4.2. Furthermore, we have, by (2.15),

$$
\begin{aligned}
\int_{0}^{\infty} H(\delta T(s, \delta u)) d s & =\delta u H\left(\delta \mu_{0}(\delta u)\right)+\int_{\delta u}^{\infty} H(\delta \mu(\delta s)) d s \\
& =: H_{1}(\delta u)+H_{2}(\delta u) .
\end{aligned}
$$

It clearly suffices to show that each term $H_{1}(u)$ and $H_{2}(u)$ satisfies (4.3) and (4.4). For $H_{2}(u)$ this was proved in the proof of Theorem 3.5. Now turn to $H_{1}(u)$. Let $\delta \in(0,1)$. Then it follows from Potter's bounds and regular variation of $\boldsymbol{\mu}$ and $H$ that for $u>1 / \delta$, say,

$$
H_{1}(\delta u) \leq C \delta^{\theta} H_{1}(u),
$$

where $\theta$ is a real constant. For small $u \leq 1 / \delta$ one can again proceed as in the proof of Theorem 3.5. Making use of the fact that $H$ is the tail of a one-dimensional Lévy measure and that $\mu(0) \geq 1$, we see that

$$
H_{1}(\delta u) \leq C[\delta u] H(\delta) \leq C[\delta u] \delta^{-2} \leq C \delta^{-1} u H\left(\mu_{0}(u)\right)=C H_{1}(u) .
$$

3.4. Integral of a nonnegative subadditive function. The functional $\phi_{\text {sojourn }}$ of Theorem 3.5 is a particular case of a more general group of subadditive functionals obtained by appropriate space-dependent weighting of the positive values of a process. Consider a nondecreasing nonnegative function $f$ such that $f(x)=0$ for $x \leq 0$ and

$$
f\left(x_{1}+x_{2}\right) \leq f\left(x_{1}\right)+f\left(x_{2}\right) \text { for } x_{1}, x_{2}>0
$$

and let

$$
\phi_{I(f)}(\boldsymbol{\alpha})=\int_{0}^{\infty} f(\alpha(t)) d t .
$$

It is clear that $\phi_{I(f)}$ is a subadditive functional. We will not address here the question of what functionals $\phi_{I(f)}$ fit in the framework of the theory developed in the present paper. Instead, we will briefly consider the class of functionals corresponding to the power functions

$$
f(x)=\left[x_{+}\right]^{p}, \quad 0 \leq p \leq 1 .
$$

We will denote the corresponding functional by $\phi_{p}(\boldsymbol{\alpha})$. The case $p=0$ corresponds to the functional $\phi_{\text {sojourn}}$.

The tail behavior of the distribution of the functional $\phi_{p}(\boldsymbol{\alpha})$ is described in the following theorem. Its proof is very similar to that of Theorem 3.5, but quite a bit longer. We omit the argument. 
THEOREM 3.7. Assume (2.5)-(2.7). Then, for every $0<p \leq 1$,

$$
\begin{aligned}
P\left(\phi_{p}(\mathbf{X}-\boldsymbol{\mu})>u\right) & =P\left(\int_{0}^{\infty}[X(t)-\mu(t)]_{+}^{p} d t>u\right) \\
& \sim C(\alpha, \beta, p) u\left(F^{\leftarrow}(u)\right)^{-p} H\left(F^{\leftarrow}(u)\right)
\end{aligned}
$$

as $u \rightarrow \infty$. Here

$$
F(x)=x^{p} \mu^{\leftarrow}(x), \quad x>0,
$$

and $C(\alpha, \beta, p)$ is a finite positive constant given by

$$
C(\alpha, \beta, p)=\int_{0}^{\infty} y(t)^{-\alpha} t^{-\alpha \beta} d t,
$$

where $y(t)=h^{-1}\left(t^{-(1+p \beta)}\right), t>0$, and $h$ is a strictly increasing continuous function on $[1, \infty)$ given by

$$
h(y)=p y^{p} \int_{1 / y}^{1}\left((y z)^{\beta}-1\right)(z-1)^{p-1} d t .
$$

3.5. The supremum of the integral of the process. Here we consider the subadditive functional

$$
\phi_{\text {supint }}(\boldsymbol{\alpha})=\sup _{v>0} \int_{0}^{v} \alpha(t) d t .
$$

Unlike other functionals considered in this section, this functional is affected by the negative values of the process. The tail behavior of this functional is described in the theorem below. Its proof, once again, is very similar to that of the previous results, but longer. We will omit its argument as well.

TheOrem 3.8. Assume (2.5)-(2.7). Then

$$
\begin{aligned}
P\left(\phi_{\text {supint }}(\mathbf{X}-\boldsymbol{\mu})>u\right) & =P\left(\sup _{v \geq 0} \int_{0}^{v}(X(t)-\mu(t)) d t>u\right) \\
& \sim C(\alpha, \beta) \mu_{1}^{\leftarrow}(u) H\left(\frac{u}{\mu_{1}^{\leftarrow}(u)}\right)
\end{aligned}
$$

as $u \rightarrow \infty$. Here

$$
\mu_{1}(x)=\int_{0}^{x} \mu(y) d y, \quad x>0,
$$

and $C(\alpha, \beta)$ is a finite positive constant given by

$$
C(\alpha, \beta)=\int_{0}^{\infty} y(t)^{-\alpha} t^{-\alpha} d t,
$$


where $y(t)=h^{-1}\left(4^{\beta}(1+\beta) t^{1+\beta}\right), t>0$, and $h$ is a strictly increasing continuous function on $[0, \infty)$ given by

$$
h(y)=\frac{y^{1+\beta}}{(1+y)^{\beta}}
$$

4. More general conditions on the function $T(s, u)$. Let $T(s, u)$ and $T(u)$ be as above. The following assumptions are needed to replace the explicit computation of the function $T(s, u)$ which we were able to perform in some of the examples above:

1. Relation between $T(s, u)$ and $T(u)$-There is $A_{1}>0$ such that

$$
T(s, u) \leq A_{1}[\mu(s)+T(u)] \text { for all } s, u>0 .
$$

2. A scaling property-There are positive functions $g(\delta)$ and $h(\delta), 0<\delta \leq 1$, satisfying

$$
h(\delta) \rightarrow 1 \quad \text { as } \delta \uparrow 1, \quad|\log (g(\delta))| \leq O\left(\delta^{-1}\right) \quad \text { as } \delta \downarrow 0
$$

and such that, for every $u>u(\delta)$ and $0<\delta \leq 1$,

$$
\int_{0}^{\infty} H(\delta T(s, \delta u)) d s \leq h(\delta) \int_{0}^{\infty} H(T(s, u)) d s,
$$

and, for every $u \geq u_{0}$ and $0<\delta \leq 1$,

$$
\int_{0}^{\infty} H(\delta T(s, \delta u)) d s \leq g(\delta) \int_{0}^{\infty} H(T(s, u)) d s .
$$

4.1. Some implications of the assumptions. We collect some particular consequences of the assumptions on the Lévy measure, the drift $\mu$ and the functional $\phi$ in Section 2 and on the function $T(s, u)$ in Section 4. All these assumptions are assumed to hold in the following lemma.

\section{LEMMA 4.1. The following statements hold:}

(i) With probability 1, for every $\gamma>0, \phi(|\mathbf{X}|-\gamma \mu)<\infty$ and therefore $\phi(\mathbf{X}-\gamma \mu)<\infty$.

(ii) For every $\varepsilon>0$ and $u>0, \gamma>0$,

$$
\begin{aligned}
\int_{0}^{\infty} & H\left(\frac{1+\varepsilon}{\gamma} T(s, u)\right) d s \\
& \leq \int_{0}^{\infty} \int_{0}^{\infty} \mathbb{1}\left(\phi\left(\gamma x \mathbb{1}_{[s, \infty)}-\boldsymbol{\mu}\right)>u\right) \rho(d x) d s \\
& \leq \int_{0}^{\infty} H\left(\frac{1}{\gamma} T(s, u)\right) d s .
\end{aligned}
$$


(iii) There is a $u_{1} \geq 0$ such that, for every $\varepsilon>0$ and $u>u_{1}$,

$$
\int_{0}^{\infty} H(\varepsilon T(s, u)) d s<\infty .
$$

(iv) For every $\gamma>0$,

$$
\begin{array}{ll}
\phi\left(\gamma \times \mathbb{1}_{[s, \infty)}-\boldsymbol{\mu}\right)<\infty & \begin{array}{l}
\text { outside a set of measure zero } \\
\text { with respect to } \rho \times \text { Leb. }
\end{array}
\end{array}
$$

(v) There exists $\beta_{1}>0$ such that, for every $u \geq 2$,

$$
H(u) \geq u^{-\beta_{1}} \text { and } \quad \mu(u) \leq u^{\beta_{1}} .
$$

(vi) There is a constant $A_{2}>0$ such that, for all $s, t \geq t_{0}$ and sufficiently large $t_{0}>0$,

$$
\mu(s+t) \leq A_{2}[\mu(s)+\mu(t)]
$$

Proof. (i) Observe first that if $\beta>\max \left(\alpha^{-1}, 0.5\right)$, we conclude from the regular variation of $H$ and $\boldsymbol{\mu}$ [see (2.5), (2.7)], and standard a.s. limit results [law of the iterated logarithm when $E[X(1)]^{2}<\infty$, generalized strong laws of large numbers when $E[X(1)]^{2}=\infty$; see Stout (1974)] that $X(t) / t^{\beta-\delta} \rightarrow 0$ a.s. as $t \rightarrow \infty$ for positive $\delta$ provided that $\beta-\delta>\max \left(0.5, \alpha^{-1}\right)$. Therefore

$$
|X(t)|-\varepsilon \mu(t) \leq|X(t)|-C t^{\beta-\delta} \rightarrow-\infty .
$$

So we may conclude from (2.9) that $\phi(|\mathbf{X}|-\varepsilon \boldsymbol{\mu})<\infty$ a.s. (with the usual convention of taking pointwise absolute values of a function).

(ii) This statement follows from the definition (2.12) of the function $T$.

(iii) Regular variation of $\boldsymbol{\mu}$ with a positive index implies the following quasimonotonicity condition: there is a $C \in(0,1]$ and $t_{0} \geq 0$ such that

$$
\inf _{s \geq t} \mu(s) \geq C \mu(t) \quad \text { for all } t \geq t_{0} .
$$

Now it follows from (2.8), (4.10) and (2.10) that $T(s, u) \geq \max (C \mu(s), T(u))$ for all $s \geq t_{0}$ and that $T(u)>0$ for every sufficiently large $u$ (say, $u>u_{1}$ ). Hence

$$
\int_{0}^{\infty} H(\varepsilon T(s, u)) d s \leq\left(t_{0}+1\right) H(\varepsilon T(u))+\int_{t_{0}+1}^{\infty} H(C \varepsilon \mu(s)) d s .
$$

The right-hand integral is finite by virtue of (2.5) and (2.7).

(iv) Relation (4.7) follows from (2.7) and (2.9).

(v) The inequalities (4.8) are immediate consequences of the regular variation property of $H$ and $\mu$.

(vi) Relation (4.9) is an immediate consequence of the regular variation of $\mu$. 
4.2. How do we verify condition (4.1)? Here is an easily verifiable sufficient condition for (4.1).

PROPOSITION 4.2. Assume that the following conditions hold:

(i) The subadditive functional $\phi$ satisfies (2.8)-(2.11).

(ii) There exists $\gamma>0$ such that, for all $0<c<1$,

$$
\phi\left(c x \mathbb{1}_{[0, \infty)}-\boldsymbol{\mu}\right) \leq c^{\gamma} \phi\left(x \mathbb{1}_{[0, \infty)}-\boldsymbol{\mu}\right) .
$$

(iii) There exists $a>0$ such that, for every $s, x>0$,

$$
\phi\left(x \mathbb{1}_{[s, \infty)}-\boldsymbol{\mu}_{s}\right) \geq \phi\left(\operatorname{ax} \mathbb{1}_{[0, \infty)}-\boldsymbol{\mu}\right),
$$

where $\mu_{s}(t)=\mu\left((t-s)_{+}\right)$.

(iv) $\boldsymbol{\mu}$ is nondecreasing.

Then (4.1) holds.

In fact, condition

$$
\phi(c \boldsymbol{\alpha}) \leq c^{\gamma} \phi(\boldsymbol{\alpha}) \text { for every } 0<c<1
$$

implies, and is more restrictive than, (2.11) and (4.11). Indeed, if (4.13) holds, monotonicity of $\phi$ implies

$$
\begin{aligned}
\phi\left(c x \mathbb{1}_{[0, \infty)}-\boldsymbol{\mu}\right) & =\phi\left(c\left(x \mathbb{1}_{[0, \infty)}-c^{-1} \boldsymbol{\mu}\right)\right) \\
& \leq c^{\gamma} \phi\left(x \mathbb{1}_{[0, \infty)}-c^{-1} \boldsymbol{\mu}\right) \leq c^{\gamma} \phi\left(x \mathbb{1}_{[0, \infty)}-\boldsymbol{\mu}\right) .
\end{aligned}
$$

Moreover, many of the functionals of interest have the property

$$
\phi\left(x \mathbb{1}_{[s, \infty)}-\boldsymbol{\mu}_{s}\right)=\phi\left(x \mathbb{1}_{[0, \infty)}-\boldsymbol{\mu}\right),
$$

which implies (4.12).

The proof of the proposition is based on the following property of the function $T(u)$.

LEMMA 4.3. There is a constant $B>0$ such that, for all $u, v>0$,

$$
T(u+v) \leq B[T(u)+T(v)] .
$$

PROOF. By monotonicity of $\phi$, for every $\varepsilon>0$,

$$
\phi\left([T(u)+T(v)+\varepsilon] \mathbb{1}_{[0, \infty)}-\mu\right) \geq \max (u, v) \geq \frac{u+v}{2},
$$

which implies that

$$
T\left(\frac{u+v}{2}\right) \leq T(u)+T(v) .
$$


Let $u>0$ and suppose that $T(2 u)>0$. By the scaling property (4.11) we have, for every $\varepsilon>0$,

$$
u>\frac{1}{2} \phi\left((1-\varepsilon) T(2 u) \mathbb{1}_{[0, \infty)}-\boldsymbol{\mu}\right) \geq \phi\left(2^{-1 / \gamma}(1-\varepsilon) T(2 u) \mathbb{1}_{[0, \infty)}-\boldsymbol{\mu}\right),
$$

which means that

$$
2^{-1 / \gamma} T(2 u) \leq T(u) .
$$

On the other hand, if $T(2 u)=0$, then this relation is trivial. The above relation, together with (4.16), yields the desired relation (4.15).

ProOF OF Proposition 4.2. It follows from (4.9) (in which we assume, without loss of generality, that $\left.A_{2} \geq 1\right), \mu(t) \leq A_{2}[\mu(t-s)+\mu(s)]$ for $s<t$, and so

$$
\boldsymbol{\mu} \mathbb{1}_{[s, \infty)} \leq A_{2}\left[\boldsymbol{\mu}_{s}+\mu(s) \mathbb{1}_{[s, \infty)}\right],
$$

implying by monotonicity of $\phi$ that

$$
r(x, s):=\phi\left(x \mathbb{1}_{[s, \infty)}-\boldsymbol{\mu} \mathbb{1}_{[s, \infty)}\right) \geq \phi\left(A_{2}\left[\left(x / A_{2}-\mu(s)\right) \mathbb{1}_{[s, \infty)}-\boldsymbol{\mu}_{s}\right]\right) .
$$

Now (2.11) and (4.12) yield

$$
\begin{aligned}
r(x, s) & \geq \phi\left(\left(x / A_{2}-\mu(s)\right) \mathbb{1}_{[s, \infty)}-\boldsymbol{\mu}_{s}\right) \\
& \geq \phi\left(a\left[x / A_{2}-\mu(s)\right] \mathbb{1}_{[0, \infty)}-\boldsymbol{\mu}\right) .
\end{aligned}
$$

Now it follows from subadditivity that

$$
\phi\left(x \mathbb{1}_{[s, \infty)}-\boldsymbol{\mu}\right) \geq \phi\left(a\left[x / A_{2}-\mu(s)\right] \mathbb{1}_{[0, \infty)}-\boldsymbol{\mu}\right)-\phi\left(\boldsymbol{\mu} \mathbb{1}_{[0, s)}\right) .
$$

Let $\varepsilon>0$ and choose $x:=(1+\varepsilon) A_{2}\left[\mu(s)+a^{-1} T(u)\right]$. Then, by (4.17),

$$
\phi\left((1+\varepsilon) A_{2}\left[\mu(s)+a^{-1} T(u)\right] \mathbb{1}_{[s, \infty)}-\boldsymbol{\mu}\right) \geq u-\left(\boldsymbol{\mu} \mathbb{1}_{[0, s)}\right),
$$

which implies that

$$
T\left(s, u-\phi\left(\mu \mathbb{1}_{[0, s)}\right)\right) \leq A_{2}\left[\mu(s)+a^{-1} T(u)\right],
$$

and, after a change of variable and with (4.15),

$$
\begin{aligned}
T(s, u) & \leq A_{2}\left[\mu(s)+a^{-1} T\left(u+\phi\left(\mu \mathbb{1}_{[0, s)}\right)\right)\right] \\
& \leq A_{2}\left[\mu(s)+a^{-1} B T(u)+a^{-1} B T\left(\phi\left(\mu \mathbb{1}_{[0, s)}\right)\right)\right] \\
& =: A_{2}\left[\mu(s)+a^{-1} B T(u)\right]+a^{-1} A_{2} B T(g(s)) .
\end{aligned}
$$

It remains to estimate the last term in (4.18). If it is nonzero, according to (2.12) we have

$$
\phi\left(\frac{1}{2} T(g(s)) \mathbb{1}_{[0, \infty)}-\boldsymbol{\mu}\right) \leq g(s) .
$$


Assume that

$$
T(g(s))>4 \mu(s) .
$$

Using (4.11), (2.8) and monotonicity of $\phi$ we obtain

$$
\begin{aligned}
\phi\left(\frac{1}{2} T(g(s)) \mathbb{1}_{[0, \infty)}-\boldsymbol{\mu}\right) & \geq \phi\left(2 \mu(s) \mathbb{1}_{[0, \infty)}-\boldsymbol{\mu}\right) \\
& \geq \phi\left(2 \mu(s) \mathbb{1}_{[0, s)}-\boldsymbol{\mu} \mathbb{1}_{[0, s)} .\right.
\end{aligned}
$$

But $2 \mu(s)-\mu(t) \geq \mu(s) \geq \mu(t)$ for $0 \leq t<s$, and so (4.20) is at least

$$
\phi\left(\boldsymbol{\mu} \mathbb{1}_{[0, s)}\right)=g(s) .
$$

However, this contradicts (4.19).

Hence $T(g(s)) \leq 4 \mu(s)$ for all $s \geq 0$, which together with (4.18) gives us (4.1).

5. Proof of Theorem 3.1. Since we have checked that the conditions (4.1), (4.3) and (4.4) are satisfied when the function $T(s, u)$ is given by one of the functions (2.13)-(2.15), it is enough to prove the theorem under the assumptions (4.1), (4.3) and (4.4).

5.1. The basic decomposition. For fixed $0<\tau<1$ and some $\delta_{0}>0$ we introduce the set

$$
B_{\tau}=\left\{x \mathbb{1}_{[s, \infty)}: \phi\left(|x| \mathbb{1}_{[s, \infty)}-\tau \boldsymbol{\mu}\right)>\delta_{0}, s \geq 0, x \in \mathbb{R}\right\} \subset \mathbb{R}^{[0, \infty)} .
$$

LEMMA 5.1. If $\delta_{0}>\max \left(u_{1}, 1\right)$ (see Lemma 4.1) then the set $B_{\tau}$ has finite Lévy measure: $v\left(B_{\tau}\right)<\infty$.

ProOF. By definition of the Lévy measure $v$ [see (2.3)] and since (2.6), (4.6) and (2.11) hold, we have

$$
\begin{aligned}
v\left(B_{\tau}\right) & =\int_{0}^{\infty} \int_{-\infty}^{\infty} \mathbb{1}\left(x \mathbb{1}_{[s, \infty)} \in B_{\tau}\right) \rho(d x) d s \\
& \leq C \int_{0}^{\infty} \int_{0}^{\infty} \mathbb{1}\left(x \mathbb{1}_{[s, \infty)} \in B_{\tau}\right) \rho(d x) d s \\
& \leq C \int_{0}^{\infty} H\left(\frac{\tau}{2} T\left(s, \delta_{0}\right)\right) d s .
\end{aligned}
$$

The right-hand expression is finite by the choice of $\delta_{0}$.

From now on $\delta_{0}$ is chosen to satisfy the assumptions of Lemma 5.1 and, hence, $\nu\left(B_{\tau}\right)<\infty$. Since $B_{\tau}$ and $B_{\tau}^{c}$ are disjoint we can decompose $\mathbf{X}$ into a sum

$$
\mathbf{X} \stackrel{\mathrm{d}}{=} \mathbf{X}_{1}+\mathbf{X}_{2}
$$


of two independent infinitely divisible processes $\mathbf{X}_{1}$ and $\mathbf{X}_{2}$ with Lévy measures $v_{1}$ and $v_{2}$, respectively, given by

$$
v_{1}(A)=v\left(A \cap B_{\tau}\right) \quad \text { and } \quad v_{2}(A)=v\left(A \cap B_{\tau}^{c}\right)
$$

for any measurable $A \subset \mathbb{R}^{[0, \infty)}$, and where the process $\mathbf{X}_{1}$ has a representation as compound Poisson sum

$$
\mathbf{X}_{1} \stackrel{\mathrm{d}}{=} \sum_{j=1}^{N} \mathbf{Y}_{j},
$$

where $\mathbf{Y}_{1}, \mathbf{Y}_{2}, \ldots$ are iid stochastic processes on $[0, \infty)$ with common law $v_{1} / v\left(B_{\tau}\right)$, independent of a Poisson random variable $N$ with mean $v\left(B_{\tau}\right)$.

The following fact will be useful in what follows.

LEMMA 5.2. For every $\gamma>0$, with probability 1 ,

$$
\phi\left(\left|\mathbf{X}_{i}\right|-\gamma \boldsymbol{\mu}\right)<\infty, \quad i=1,2 .
$$

Proof. Since $P\left(\mathbf{X}_{1}=0\right)>0$ and $\mathbf{X}_{1}, \mathbf{X}_{2}$ are independent, it follows from Lemma 4.1(i) that $\phi\left(\left|\mathbf{X}_{2}\right|-\gamma \boldsymbol{\mu}\right)<\infty$ a.s. for every $\gamma>0$. In turn, exploiting the monotonicity and subadditivity of $\phi$, we conclude that $\phi\left(\left|\mathbf{X}_{1}\right|-\gamma \boldsymbol{\mu}\right)<\infty$ a.s. for every $\gamma>0$.

5.2. The upper bound. By (5.2) and subadditivity of $\phi$, for every $\varepsilon \in(0,1)$,

$$
\begin{aligned}
P(\phi & (\mathbf{X}-\boldsymbol{\mu})>u) \\
& =P\left(\phi\left(\mathbf{X}_{1}+\mathbf{X}_{2}-\boldsymbol{\mu}\right)>u\right) \\
& \leq P\left(\phi\left(\mathbf{X}_{1}-(1-\varepsilon) \boldsymbol{\mu}\right)>(1-\varepsilon) u\right)+P\left(\phi\left(\mathbf{X}_{2}-\varepsilon \boldsymbol{\mu}\right)>\varepsilon u\right) \\
& =: I_{1}(u)+I_{2}(u) .
\end{aligned}
$$

LEMMA 5.3. Under the assumptions of the theorem for every $\tau$ small enough,

$$
\begin{aligned}
& \lim _{\varepsilon \rightarrow 0} \limsup _{u \rightarrow \infty} I_{1}(u) / \psi(u) \leq 1, \\
& \lim _{\varepsilon \rightarrow 0} \limsup _{u \rightarrow \infty} I_{2}(u) / \psi(u)=0 .
\end{aligned}
$$

From Lemma 5.3 and (5.4) we conclude that

$$
\limsup _{u \rightarrow \infty} \frac{P(\phi(\mathbf{X}-\boldsymbol{\mu})>u)}{\psi(u)} \leq 1 .
$$

This concludes the proof of the upper bound in (3.1). 
We proceed with the proof of Lemma 5.3.

PROOF OF (5.5). The compound Poisson representation (5.3), subadditivity of $\phi$ and the same argument as in Lemma 2.7 in Mikosch and Samorodnitsky (2000) yield that

$$
\begin{aligned}
I_{1}(u) \leq & E N P\left(\phi\left(\mathbf{Y}_{1}-(1-\varepsilon)^{2} \boldsymbol{\mu}\right)>(1-\varepsilon)^{2} u\right) \\
& +\sum_{k=2}^{\infty} k^{2} P(N=k)\left[P\left(\phi\left(\mathbf{Y}_{1}-\frac{\varepsilon(1-\varepsilon)}{k} \boldsymbol{\mu}\right)>\frac{\varepsilon(1-\varepsilon)}{k} u\right)\right]^{2} \\
= & I_{11}(u)+I_{12}(u) .
\end{aligned}
$$

Recalling that $E N=v\left(B_{\tau}\right)$ and using the monotonicity properties (2.10) and (2.11) of $\phi$, we see that

$$
\begin{aligned}
I_{11}(u) & =\int_{0}^{\infty} \int_{0}^{\infty} \mathbb{1}\left(\phi\left(x \mathbb{1}_{[s, \infty)}-(1-\varepsilon)^{2} \boldsymbol{\mu}\right)>(1-\varepsilon)^{2} u\right) \rho(d x) d s \\
& \leq \int_{0}^{\infty} \int_{0}^{\infty} \mathbb{1}\left(\phi\left((1-\varepsilon)^{-2} x \mathbb{1}_{[s, \infty)}-\boldsymbol{\mu}\right)>(1-\varepsilon)^{2} u\right) \rho(d x) d s .
\end{aligned}
$$

Write, for $\varepsilon \in(0,1)$,

$$
\widetilde{h}(\varepsilon)=h\left((1-\varepsilon)^{2} /(1+\varepsilon)\right) .
$$

The function $H$ is decreasing, while $T(s, u)$ is increasing in both arguments. Therefore and in view of (4.4) we can further bound $I_{11}(u)$ as follows for sufficiently large $u$ :

$$
\begin{aligned}
I_{11}(u) & \leq \int_{0}^{\infty} H\left((1-\varepsilon)^{2} T\left(s,(1-\varepsilon)^{2} u\right)\right) d s \\
& \leq \widetilde{h}(\varepsilon) \int_{0}^{\infty} H((1+\varepsilon) T(s,(1+\varepsilon) u)) d s \\
& \leq \widetilde{h}(\varepsilon) \int_{0}^{\infty} H((1+\varepsilon) T(s, u)) d s \leq \widetilde{g}(\varepsilon) \psi(u) .
\end{aligned}
$$

In the last step we used (4.5). Similar arguments and the assumptions on the function $g$ in (4.2) yield for sufficiently large $u$ and sufficiently small $\varepsilon$ that

$$
I_{12}(u) \leq[E N]^{-2} \sum_{k=2}^{\infty} k^{2} P(N=k)\left[g\left(\frac{\varepsilon(1-\varepsilon)}{(1+\varepsilon) k}\right)\right]^{2}[\psi(u)]^{2} \leq C[\psi(u)]^{2} .
$$

Since, by (4.2), $\widetilde{h}(\varepsilon) \rightarrow 1$ as $\varepsilon \rightarrow 0$, we finally conclude that

$$
\lim _{\varepsilon \rightarrow 0} \limsup _{u \rightarrow \infty} I_{1}(u) / \psi(u) \leq \lim _{\varepsilon \rightarrow 0} \tilde{h}(\varepsilon)=1 .
$$


Proof of (5.6). Let $\widetilde{\mathbf{X}}_{2}$ be an independent copy of $\mathbf{X}_{2}$. Consider the symmetrization of $\mathbf{X}_{2}$, given by

$$
\mathbf{Z}=\mathbf{X}_{2}-\widetilde{\mathbf{X}}_{2}
$$

By independence of $\mathbf{X}_{2}$ and $\widetilde{\mathbf{X}}_{2}$ and subadditivity of $\phi$, we have

$$
P(\phi(\mathbf{Z}-\varepsilon \boldsymbol{\mu} / 2)>\varepsilon u / 2) \geq P\left(\phi\left(\mathbf{X}_{2}-\varepsilon \boldsymbol{\mu}\right)>\varepsilon u\right) P\left(\phi\left(\tilde{\mathbf{X}}_{2}-\varepsilon \boldsymbol{\mu} / 2\right) \leq \varepsilon u / 2\right) .
$$

By Lemma 5.2, the second factor on the right-hand side goes to 1 as $u \rightarrow \infty$. Hence (5.6) follows once we have proved that for every $\varepsilon>0$ and $\tau$ small enough

$$
\lim _{u \rightarrow \infty} \frac{P(\phi(\mathbf{Z}-\varepsilon \boldsymbol{\mu})>\varepsilon u)}{\psi(u)}=0 .
$$

Observe that $\mathbf{Z}$ is a symmetric infinitely divisible process whose Lévy measure $v_{Z}$ is given by

$$
v_{Z}(A)=\int_{0}^{\infty} \int_{-\infty}^{\infty}\left[\mathbb{1}\left(x \mathbb{1}_{[s, \infty)} \in A \cap B_{\tau}\right)+\mathbb{1}\left(x \mathbb{1}_{[s, \infty)} \in(-A) \cap B_{\tau}\right)\right] \rho(d x) d s
$$

$$
=\int_{0}^{\infty} \int_{-\infty}^{\infty} \mathbb{1}\left(x \mathbb{1}_{[s, \infty)} \in A \cap B_{\tau}\right) \rho^{*}(d x) d s
$$

for any measurable $A \subset \mathbb{R}^{[0, \infty)}$. Here

$$
\rho^{*}((t, \infty))=\rho((t, \infty))+\rho((-\infty,-t)), \quad t>0,
$$

is the symmetrized one-dimensional Lévy measure of $\mathbf{X}$. Define two symmetric one-dimensional Lévy measures $\rho_{1}$ and $\rho_{2}$ by

$$
\rho_{1}(\cdot)=\rho^{*}(\cdot \cap\{x:|x| \geq 1\}) \text { and } \rho_{2}(\cdot)=\rho^{*}(\cdot \cap\{x:|x|<1\}) .
$$

Let $\mathbf{Z}_{1}, \mathbf{Z}_{2}$ be independent infinitely divisible processes with Lévy measures that are obtained by replacing $\rho$ in (5.9) by $\rho_{1}, \rho_{2}$, respectively. Then

$$
\mathbf{Z} \stackrel{\mathrm{d}}{=} \mathbf{Z}_{1}+\mathbf{Z}_{2}
$$

and therefore subadditivity of $\phi$ implies

$$
\begin{aligned}
P(\phi(\mathbf{Z}-\varepsilon \boldsymbol{\mu})>\varepsilon u) & \leq P\left(\phi\left(\mathbf{Z}_{1}-\varepsilon \boldsymbol{\mu} / 2\right)>\varepsilon u / 2\right)+P\left(\phi\left(\mathbf{Z}_{2}-\varepsilon / 2 \boldsymbol{\mu}\right)>\varepsilon u / 2\right) \\
& =: I_{3}(u)+I_{4}(u) .
\end{aligned}
$$

It follows from Lemmas 6.1 and 6.2 below that, for any $\varepsilon>0$ and $\tau>0$,

$$
\lim _{u \rightarrow \infty} I_{4}(u) / \psi(u)=0,
$$

and so we proceed to estimate $I_{3}(u)$. 
Let $\left(\Gamma_{j}\right)$ be the points of a time homogeneous Poisson process on $[0, \infty)$ with rate $\lambda=\rho_{1}(\mathbb{R})$, and let $\left(V_{j}\right)$ be iid symmetric random variables with common distribution $\rho_{1} / \rho_{1}(\mathbb{R})$ and independent of the Poisson process. Define, for $j \geq 1$,

$$
Y_{j}=V_{j} \mathbb{1}\left(\phi\left(\left|V_{j}\right| \mathbb{1}_{\left[\Gamma_{j}, \infty\right)}-\tau \boldsymbol{\mu}\right) \leq \delta_{0}\right) .
$$

Observe that we can represent the process $\mathbf{Z}_{1}$ in the form

$$
Z_{1}(t)=\sum_{j=1}^{\infty} Y_{j} \mathbb{1}_{\left[\Gamma_{j}, \infty\right)}(t), \quad t \geq 0 .
$$

(Simply compute the mean measures of the Poisson random measures on both sides of the equation above.) Notice that

$$
\left(\Gamma_{1}, \pm Y_{1}, \Gamma_{2}, \pm Y_{2}, \ldots\right) \stackrel{\mathrm{d}}{=}\left(\Gamma_{1}, Y_{1}, \Gamma_{2}, Y_{2}, \ldots\right)
$$

for any choice of signs above.

For $u>0$ and $T(u)=T(0, u)$ let

$$
m=m(u)=\inf \{j=0,1,2, \ldots: \mu(2 j) \geq T(u)\}
$$

and

$$
\mathbf{Z}_{1,(m)}=\sum_{j=1}^{m} Y_{j} \mathbb{1}_{\left[\Gamma_{j}, \infty\right)} \quad \text { and } \quad \mathbf{Z}_{1}^{(m)}=\sum_{j=m+1}^{\infty} Y_{j} \mathbb{1}_{\left[\Gamma_{j}, \infty\right)} .
$$

Then, again by subadditivity,

$$
I_{3}(u) \leq P\left(\phi\left(\mathbf{Z}_{1,(m)}-\varepsilon \boldsymbol{\mu} / 4\right)>\varepsilon u / 4\right)+P\left(\phi\left(\mathbf{Z}_{1}^{(m)}-\varepsilon \boldsymbol{\mu} / 4\right)>\varepsilon u / 4\right) .
$$

However, the right-hand expressions are of the order $o(\psi(u))$ as $u \rightarrow \infty$ for every $\varepsilon>0$ and $\tau>0$ small enough (relative to $\varepsilon$ ), as follows from Lemmas 6.4 and 6.6. This and (5.12) imply (5.8) and complete the proof of (5.6).

5.3. The lower bound. We again start with the identity

$$
P(\phi(\mathbf{X}-\boldsymbol{\mu})>u)=P\left(\phi\left(\mathbf{X}_{1}+\mathbf{X}_{2}-\boldsymbol{\mu}\right)>u\right) .
$$

Recalling that $\mathbf{X}_{1}$ and $\mathbf{X}_{2}$ are independent and $\phi$ is subadditive, for every $K>0$,

$$
\begin{aligned}
& P(\phi(\mathbf{X}-\boldsymbol{\mu})>u) \\
& \quad \geq P\left(\phi\left(\mathbf{X}_{1}-(1+\varepsilon) \boldsymbol{\mu}\right)>u+K\right) P\left(\phi\left(-\mathbf{X}_{2}-\varepsilon \boldsymbol{\mu}\right) \leq K\right) \\
& \quad=I_{5}(u, K) I_{6}(K) .
\end{aligned}
$$

LEMMA 5.4. Under the assumptions of the theorem,

$$
\lim _{K \rightarrow \infty} \liminf _{u \rightarrow \infty} I_{5}(u, K) / \psi(u) \geq 1 .
$$


It is immediate from Lemma 5.2 that $\lim _{K \rightarrow \infty} I_{6}(K)=1$. Therefore, from this lemma and (5.18) we conclude that

$$
\liminf _{u \rightarrow \infty} \frac{P(\phi(\mathbf{X}-\boldsymbol{\mu})>u)}{\psi(u)} \geq 1 .
$$

This completes the proof of the lower bound in (3.1).

Proof of Lemma 5.4. First recall the compound Poisson structure of $\mathbf{X}_{1}$ from (5.3). For $k=1,2, \ldots$ and $j \leq k$, consider the disjoint events

$$
\begin{aligned}
& B_{k j}=\left\{N=k, \phi\left(\mathbf{Y}_{j}-(1+\varepsilon)^{2} \boldsymbol{\mu}\right)\right.>u+2 K, \\
& \phi\left(\mathbf{Y}_{i}-(1+\varepsilon)^{2} \boldsymbol{\mu}\right) \leq u+2 K, i=1, \ldots, k, i \neq j, \\
&\left.\phi\left(-\sum_{1 \leq i \neq j \leq k} \mathbf{Y}_{i}-\varepsilon(1+\varepsilon) \boldsymbol{\mu}\right) \leq K\right\} .
\end{aligned}
$$

Subadditivity of $\phi$ implies

$$
\begin{aligned}
I_{5}(u, K) & \geq \sum_{k=1}^{\infty} \sum_{j=1}^{k} P\left(B_{k j}\right) \\
& \geq P\left(\phi\left(\mathbf{Y}_{1}-(1+\varepsilon)^{2} \boldsymbol{\mu}\right)>u+2 K\right) p_{1}(K)-p_{2}(u),
\end{aligned}
$$

where

$$
\begin{gathered}
p_{1}(K):=\sum_{k=1}^{\infty} \sum_{j=1}^{k} P\left(N=k, \phi\left(-\sum_{1 \leq i \neq j \leq k} \mathbf{Y}_{i}-\varepsilon(1+\varepsilon) \boldsymbol{\mu}\right) \leq K\right), \\
p_{2}(u):=\sum_{k=2}^{\infty} P\left(N=k, \phi\left(\mathbf{Y}_{j}-(1+\varepsilon)^{2} \boldsymbol{\mu}\right)>u+2 K\right. \\
\quad \text { for at least } 2 \text { different } j \in\{1, \ldots, k\}) .
\end{gathered}
$$

Using the independence of $\mathbf{Y}_{j}$ and $\sum_{1 \leq i \neq j \leq k} \mathbf{Y}_{i}$ and again the subadditivity of $\phi$, we have

$p_{1}(K) \geq E N P\left(\phi\left(\mathbf{Y}_{1}-\varepsilon(1+\varepsilon) \boldsymbol{\mu} / 2\right) \leq K / 2\right) P\left(\phi\left(-\mathbf{X}_{1}-\varepsilon(1+\varepsilon) \boldsymbol{\mu} / 2\right) \leq K / 2\right)$.

By Lemma 5.2, $\phi\left(\left|\mathbf{X}_{1}\right|-\gamma \boldsymbol{\mu}\right)<\infty$ a.s. for every $\gamma>0$. Using this fact, (2.10) and (4.7) [recall that the law of $\mathbf{Y}_{1}$ is $v_{1} / v\left(B_{\tau}\right)$, see (5.3)] we see that

$$
\liminf _{K \rightarrow \infty} p_{1}(K) / E N \geq 1 \text {. }
$$

The argument leading to (5.5) also shows that, for every $K>0$,

$$
\liminf _{u \rightarrow \infty} \frac{E N P\left(\phi\left(\mathbf{Y}_{1}-(1+\varepsilon)^{2} \boldsymbol{\mu}\right)>u+2 K\right)}{\psi(u)} \geq \frac{1}{h\left((1+\varepsilon)^{-3}\right)},
$$


implying that

$$
\begin{aligned}
& \lim _{\varepsilon \rightarrow 0} \lim _{K \rightarrow \infty} \liminf _{u \rightarrow \infty} \frac{p_{1}(K) P\left(\phi\left(\mathbf{Y}_{1}-(1+\varepsilon)^{2} \boldsymbol{\mu}\right)>u+2 K\right)}{\psi(u)} \\
& \quad \geq \lim _{\varepsilon \rightarrow 0} \frac{1}{h\left((1+\varepsilon)^{-3}\right)}=1 .
\end{aligned}
$$

Since, as $u \rightarrow \infty$,

$$
p_{2}(u) \leq E\left(N^{2}\right)\left[P\left(\phi\left(\mathbf{Y}_{1}-(1+\varepsilon)^{2} \boldsymbol{\mu}\right)>u+2 K\right)\right]^{2},
$$

(5.19) follows.

6. Auxiliary facts and lemmas. In this section we provide some auxiliary results for the proof of Theorem 3.1. In what follows, we always assume that the assumptions of this theorem are satisfied.

We start with a simple lemma connecting the rates of decay of the function $\psi(u)$ in (2.4) and of $1 / T(u)$ in (2.12).

LEMMA 6.1. There are constants $q>0$ and $C>0$ such that, for all $u \geq 1$,

$$
\psi(u) \geq C[T(u)]^{-q} .
$$

Proof. Recalling the definition of $\psi(u)$ from (2.4), observing that $H$ is decreasing and using (4.1), we obtain

$$
\psi(u) \geq \int_{0}^{\infty} H(2 T(s, u)) d s \geq \int_{0}^{\infty} H\left(2 A_{1}[\mu(s)+T(u)]\right) d s .
$$

Now use that both $\mu(s)$ and $H(u)$ can be bounded by power laws [see (4.8)], and change the variable of integration to get the desired result.

Next we discuss certain properties of the processes occurring in (5.2) and subsequent decompositions. The processes $\mathbf{X}_{i}$ have independent (though not necessarily stationary) increments. This property is inherited by the symmetric processes $\mathbf{Z}$ in (5.7) and, subsequently, $\mathbf{Z}_{i}$ in (5.11).

Let $\mathbf{W}_{i}$ be Lévy processes on $[0, \infty)$ with one-dimensional Lévy measure $\rho_{i}$, $i=1,2$, as defined in (5.10). Then the following identities in law hold:

$$
\mathbf{W}_{i} \stackrel{\mathrm{d}}{=} \mathbf{Z}_{i}+\mathbf{V}_{i}, \quad i=1,2,
$$

where, for fixed $i, \mathbf{Z}_{i}$ and $\mathbf{V}_{i}$ are independent symmetric infinitely divisible processes with independent increments.

Our next lemma shows that the functional $\phi$ applied to the process $\mathbf{Z}_{2}$, representing the "small jumps" of the process $\mathbf{Z}$, has a "light" tailed distribution. 
LEMMA 6.2. For every $\gamma>0$ and $r>0$, we have

$$
J(u):=P\left(\phi\left(\mathbf{Z}_{2}-\gamma \boldsymbol{\mu}\right)>u\right)=o\left([T(u / 2)]^{-r}\right) \quad \text { as } u \rightarrow \infty .
$$

PROOF. Without loss of generality we may assume that $r \geq 2$ and $\gamma \leq 1$. By subadditivity of $\phi$,

$$
\begin{aligned}
J(u) \leq & P\left(\phi\left(\mathbf{Z}_{2} \mathbb{1}_{[0, T(u / 2))}-\gamma \boldsymbol{\mu} / 2\right)>u / 2\right) \\
& +P\left(\phi\left(\mathbf{Z}_{2} \mathbb{1}_{[T(u / 2), \infty)}-\gamma \boldsymbol{\mu} / 2\right)>u / 2\right) \\
= & : J_{1}(u)+J_{2}(u) .
\end{aligned}
$$

The monotonicity properties (2.10), (2.11) of $\phi$ yield

$$
\begin{aligned}
J_{1}(u) & \leq P\left(\phi\left(\sup _{0 \leq t \leq T(u / 2)} Z_{2}(t) \mathbb{1}_{[0, T(u / 2))}-\gamma \mu / 2\right)>u / 2\right) \\
& \leq P\left(\sup _{0 \leq t \leq T(u / 2)} Z_{2}(t) \geq \gamma T(u / 2) / 2\right) \\
& \leq 2 P\left(Z_{2}(T(u / 2)) \geq \gamma T(u / 2) / 2\right) .
\end{aligned}
$$

In the last step we used Lévy's maximal inequality. Another appeal to this inequality and to the definition of $\mathbf{W}_{2}$ in (6.2) gives

$$
J_{1}(u) \leq 4 P\left(W_{2}(T(u / 2)) \geq \gamma T(u / 2) / 2\right) .
$$

However, the Lévy process $\mathbf{W}_{2}$ has a symmetric Lévy measure supported by a compact set. Therefore, it has finite exponential moments. By the BurkholderGundy inequality, for every $p \geq 2$ there is a positive constant $C$ such that $E\left|W_{2}(t)\right| \leq C t^{p / 2}$ for all $t>0$. Applying Markov's inequality, we finally obtain the following bound:

$$
J_{1}(u)=o\left([T(u / 2)]^{-r}\right), \quad u \rightarrow \infty .
$$

Now we turn to the estimation of $J_{2}(u)$. We proceed in a similar fashion. First, monotonicity of $\phi$ together with (2.8) gives

$$
\begin{aligned}
J_{2}(u) \leq & P\left(\phi\left(\left(\mathbf{Z}_{2}-\gamma \mu / 2\right) \mathbb{1}_{[T(u / 2), \infty)}\right)>u / 2\right) \\
\leq & P\left(Z_{2}(t)>\gamma \mu(t) / 2 \text { for some } t \geq T(u / 2)\right) \\
\leq & \sum_{j=1}^{\infty} P\left(Z_{2}(t)>\gamma \mu(t) / 2\right. \\
& \quad \text { for some } T(u / 2)+(j-1) \leq t<T(u / 2)+j)=: \sum_{j=1}^{\infty} b_{j} .
\end{aligned}
$$


Now use again Lévy's maximal inequality and the fact that $\boldsymbol{\mu}$ is quasimonotone and converges to infinity to obtain the following chain of inequalities:

$$
\begin{aligned}
b_{j} & \leq 2 P\left(Z_{2}(T(u / 2)+j)>C \gamma \mu(T(u / 2)+(j-1)) / 2\right) \\
& \leq 4 P\left(W_{2}(T(u / 2)+j)>C \gamma \mu(T(u / 2)+(j-1)) / 2\right) .
\end{aligned}
$$

By virtue of the regular variation condition on $\mu$, there are $C>0$ and $\beta>\beta_{2}>$ $\max \left(\alpha^{-1}, 0.5\right)$ such that

$$
\mu(t) \geq C t^{\beta_{2}}, \quad t>0 .
$$

Finally, applying the Burkholder-Gundy and Markov inequalities and choosing $p>2\left(\beta_{2}+r\right) / \beta_{2}$, we obtain, for any $r \geq 2$,

$$
J_{2}(u) \leq C \sum_{j=1}^{\infty} \frac{[T(u / 2)+j]^{p / 2}}{[\mu(T(u / 2)+(j-1))]^{p}}=o\left([T(u / 2)]^{-r}\right) .
$$

The latter estimate together with (6.4) for $J_{1}(u)$ establishes the desired bound (6.3) for $J(u)$.

Now we turn to the processes $\mathbf{Z}_{1,(m)}$ and $\mathbf{Z}_{1}^{(m)}$ defined in (5.16). In this context, recall that $\left(Y_{j}\right)$ is a sequence of independent symmetric random variables given the points $\left(\Gamma_{k}\right)$ of a homogeneous Poisson process with rate $\lambda=\rho_{1}(\mathbb{R})$. Write

$$
\begin{aligned}
A_{m} & =\left\{\left|Y_{j}\right| \leq \theta T\left(\Gamma_{j}, u\right), j=1, \ldots, m\right\}, \quad m \geq 1, \\
S_{k} & =Y_{1}+\cdots+Y_{k}, \quad k \geq 1 .
\end{aligned}
$$

LEMMA 6.3. Let $m=m(u)$ be defined by (5.15). For every $\gamma>0$ and $r>0$, there are positive constants $\theta$ and $C$ such that, for all $u>0$,

$$
G(u):=P\left(\phi\left(\mathbf{Z}_{1,(m)}-\gamma \boldsymbol{\mu}\right)>u, A_{m}\right) \leq C m^{-r} .
$$

PROOF. Without loss of generality assume that $\gamma \leq 1$. By monotonicity of $\phi$,

$$
\begin{aligned}
\phi\left(\sum_{j=1}^{m} Y_{j} \mathbb{1}_{\left[\Gamma_{j}, \infty\right)}-\gamma \boldsymbol{\mu}\right) & \leq \phi\left(\max _{1 \leq k \leq m} S_{k} \mathbb{1}_{\left[\Gamma_{1}, \infty\right)}-\gamma \boldsymbol{\mu}\right) \\
& \leq \phi\left(\gamma^{-1} \max _{1 \leq k \leq m} S_{k} \mathbb{1}_{\left[\Gamma_{1}, \infty\right)}-\boldsymbol{\mu}\right) .
\end{aligned}
$$

Therefore we have

$$
\begin{aligned}
G(u) & \leq P\left(\max _{1 \leq k \leq m} S_{k} \geq \gamma T\left(\Gamma_{1}, u\right), A_{m}\right) \\
& \leq P\left(\max _{1 \leq k \leq m} S_{k} \geq \gamma T\left(\Gamma_{1}, u\right), A_{m}, \Gamma_{m} \leq 2 \lambda m\right)+P\left(\Gamma_{m}>2 \lambda m\right) \\
& =: p_{m}^{(1)}+p_{m}^{(2)} .
\end{aligned}
$$


Obviously, $p_{m}^{(2)}$ decays to zero at an exponential rate. As to $p_{m}^{(1)}$, observe that

$$
\left\{A_{m}, \Gamma_{m} \leq 2 \lambda m\right\} \subset\left\{\max _{j=1, \ldots, m}\left|Y_{j}\right| \leq \theta T(2 \lambda m, u)\right\}=: \widetilde{A}_{m} .
$$

Therefore and by virtue of Lévy's maximal inequality, applied conditonally upon $\left(\Gamma_{k}\right)$ and $\left(\left|Y_{k}\right|\right)$,

$p_{m}^{(1)} \leq P\left(\max _{1 \leq k \leq m} S_{k} \geq \gamma T(u), \widetilde{A}_{m}\right) \leq 2 P\left(S_{m} \geq \gamma T(u), \widetilde{A}_{m}\right) \leq 4 P\left(\widetilde{S}_{m} \geq \gamma T(u)\right)$.

In the last step we applied the contraction principle for sums of independent symmetric random variables. Here

$$
\widetilde{S}_{m}=\sum_{j=1}^{m} \tilde{Y}_{j}, \quad \tilde{Y}_{j}=\widetilde{Y}_{j}^{(m)}=Y_{j} \mathbb{1}_{\left|Y_{j}\right| \leq \theta T(2 \lambda m, u)}, \quad j=1, \ldots, m .
$$

Notice that, conditionally upon $\left(\Gamma_{k}\right), \widetilde{S}_{m}$ is a sum of independent symmetric random variables which are uniformly bounded by $T(2 \lambda m, u)$. An application of Prokhorov's exponential inequality [see Prokhorov (1959); cf. Petrov (1995), 2.6.1 on page 77], conditionally on $\left(\Gamma_{k}\right)$, yields

$$
p_{m}^{(1)} \leq 4 E \exp \left\{-\frac{\gamma T(u)}{2 \theta T(2 \lambda m, u)} \operatorname{arcsinh}\left[\frac{\theta T(2 \lambda m, u) \gamma T(u)}{2 \operatorname{var}\left(\widetilde{S}_{m} \mid\left(\Gamma_{k}\right)\right)}\right]\right\} .
$$

Let us consider the case $\alpha<2$ in (2.5); the case $\alpha \geq 2$ is analogous. With $\beta_{2}$ as in (6.5) define $\beta_{3}=2 \beta_{2} /\left(1+\alpha \beta_{2}\right)$; note that $\beta_{2}>\beta_{3}^{-1}$ and

$$
H(u)=o\left(u^{-\beta_{3}}\right), \quad u \rightarrow \infty .
$$

It follows from the representation (5.13) of the random variables $Y_{j}$ that there is a constant $C$ such that, for any realization $\left(\Gamma_{i}\right)$,

$$
\operatorname{var}\left(\widetilde{S}_{m} \mid\left(\Gamma_{k}\right)\right) \leq C m[T(2 \lambda m, u)]^{2-\beta_{3}} .
$$

Then use property (4.1) of $T(2 \lambda m, u)$ and the definition (5.15) of $m=m(u)$ to obtain

$$
T(2 \lambda m, u) \leq A_{1}[\mu(2 \lambda m)+T(u)] \leq C[\mu(2 \lambda m)+\mu(2 m)] \leq C \mu(m) .
$$

Similarly,

$$
T(2 \lambda m, u) T(u) \geq[T(u)]^{2} \geq[\mu(2(m-1))]^{2} \geq C[\mu(m)]^{2} .
$$

Combining the latter estimates and using the regular variation (2.7) on $\boldsymbol{\mu}$ with its implication (6.5), we arrive at the bound

$$
\frac{\theta T(2 \lambda m, u) \gamma T(u)}{2 \operatorname{var}\left(\widetilde{S}_{m} \mid\left(\Gamma_{k}\right)\right)} \geq C \frac{[\mu(m)]^{\beta_{3}}}{m} \geq C m^{\beta_{3} \beta_{2}-1} .
$$


Since $\beta_{2}>\beta_{3}^{-1}$, the power of $m$ is positive. Similarly,

$$
\frac{T(u)}{T(2 \lambda m, u)} \geq C \frac{T(u)}{\mu(2 \lambda m)+T(u)},
$$

which is bounded away from 0 by $(5.15)$. Using the fact that $\operatorname{arcsinh}(t) \geq$ $\log (1+t), t>0$, we conclude that

$$
p_{m}^{(1)} \leq C \exp \left\{-\frac{\log m}{C \theta}\right\} .
$$

Collecting all bounds above for $G(u), p_{m}^{(1)}$ and $p_{m}^{(2)}$, we obtain our claim by choosing $\theta$ small enough.

Our next lemma shows that the first probability on the right-hand side of (5.17) is much smaller than $\psi(u)$.

LeMmA 6.4. Let $m=m(u)$ be defined by (5.15). For every $\varepsilon>0$ and $\tau>0$ small enough (relative to $\varepsilon$ ),

$$
\lim _{u \rightarrow \infty} \frac{P\left(\phi\left(\mathbf{Z}_{1,(m)}-\varepsilon \boldsymbol{\mu}\right)>\varepsilon u\right)}{\psi(u)}=0 .
$$

Proof. Since $\mu(t)$ does not grow faster than a power function, it follows from the definition of $m=m(u)$ that there exist positive constants $C, q$ such that $m \geq C[T(u)]^{q}$ for large $u$. By virtue of Lemma 6.3, for every $r>0$ and $\varepsilon>0$ there are positive $\theta$ and $C$ such that

$$
P\left(\phi\left(\mathbf{Z}_{1,(m)}-\varepsilon \boldsymbol{\mu}\right)>\varepsilon u, A_{m}\right) \leq C m^{-r} \leq C[T(u)]^{-r q} .
$$

Since $r>0$ can be chosen arbitrarily large, the latter fact in combination with Lemma 6.1 implies that

$$
P\left(\phi\left(\mathbf{Z}_{1,(m)}-\varepsilon \boldsymbol{\mu}\right)>\varepsilon u, A_{m}\right)=o(\psi(u)) .
$$

On the other hand, we have

$$
P\left(A_{m}^{c}\right) \leq \sum_{j=1}^{m} P\left(\left|Y_{j}\right|>\theta T\left(\Gamma_{j}, u\right)\right)=2 \sum_{j=1}^{m} P\left(Y_{j}>\theta T\left(\Gamma_{j}, u\right)\right) .
$$

By representation (5.13) for the $Y_{j}$ 's,

$$
Y_{j} \leq \tau T\left(\Gamma_{j}, \delta_{0}\right) \leq \tau T\left(\Gamma_{j}, u\right)
$$

for $u$ large enough. Thus, for $\tau$ small enough, the right-hand expression in (6.7) vanishes. This concludes the proof.

The next lemmas are related to the behavior of the second term on the right-hand side of (5.17). Write

$$
A_{m n}=\left\{\left|Y_{j}\right| \leq \theta T\left(\Gamma_{j}, u\right), m<j<n+1\right\}, \quad n=m, m+1, \ldots, \infty .
$$


LEMMA 6.5. For any $\gamma>0$ and $r>0$, there are positive constants $\theta$ and $C$ such that, for all $u>0$ and $n>m=m(u)$,

$$
H_{n}:=P\left(S_{n}-S_{m}>\gamma \mu\left(\Gamma_{n}\right), A_{m n}\right) \leq C n^{-r} .
$$

PROOF. For all $j$ and $u>0$, we have $T\left(\Gamma_{j}, u\right) \leq A_{1}\left[\mu\left(\Gamma_{j}\right)+T(u)\right] \leq$ $C\left[\mu\left(\Gamma_{n}\right)+T(u)\right]$. Now, the definition of $m=m(u)$ and regular variation give, for $n>m, T(u) \leq \mu(2 m) \leq C \mu(2 n) \leq C \mu(n)$. Therefore

$$
T\left(\Gamma_{j}, u\right) \leq C\left[\mu\left(\Gamma_{n}\right)+\mu(n)\right], \quad j \leq n .
$$

Then, for $m<n$,

$$
H_{n} \leq P\left(S_{n}-S_{m}>\gamma \mu\left(\Gamma_{n}\right), \max _{j=m+1, \ldots, n}\left|Y_{j}\right| \leq \theta C\left[\mu\left(\Gamma_{n}\right)+\mu(n)\right]\right) .
$$

Recalling that the Poisson process $\left(\Gamma_{j}\right)$ has rate $\lambda$, write

$$
D_{n}=\left\{\left|\Gamma_{n}-\lambda n\right| \leq 0.5 \lambda n\right\}
$$

and notice that $P\left(D_{n}^{c}\right)$ decays to zero at an exponential rate. Therefore, for any $r>0$,

$$
\begin{aligned}
H_{n} \leq & P\left(S_{n}-S_{m}>\mu\left(\Gamma_{n}\right), \max _{l=m+1, \ldots, n}\left|Y_{j}\right| \leq \theta C\left[\mu\left(\Gamma_{n}\right)+\mu(n)\right], D_{n}\right) \\
& +P\left(D_{n}^{c}\right) \\
\leq & P\left(S_{n}-S_{m}>C^{-1} \mu(n), \max _{j=m+1, \ldots, n}\left|Y_{j}\right| \leq \theta C \mu(n)\right)+C n^{-r} \\
\leq & 2 P\left(\widehat{S}_{n}-\widehat{S}_{m} \geq C^{-1} \mu(n)\right)+C n^{-r} \\
\leq & 4 P\left(\widehat{S}_{n} \geq C^{-1} \mu(n)\right)+C n^{-r} .
\end{aligned}
$$

In the last step we used the contraction principle and Lévy's maximal inequality for the sum of conditionally independent and symmetric random variables $Y_{j}$. Here

$$
\widehat{S}_{n}=\sum_{j=1}^{n} \widehat{Y}_{j}, \quad \widehat{Y}_{j}=Y_{j} \mathbb{1}_{\left|Y_{j}\right| \leq \theta C \mu(n)}, \quad j=1, \ldots, n .
$$

Again using Prokhorov's inequality, conditionally on $\left(\Gamma_{k}\right)$ we can bound the tail probability in (6.10) by

$$
E \exp \left\{-\frac{C^{-1} \mu(n)}{2 \theta C \mu(n)} \operatorname{arcsinh}\left[\frac{\theta[\mu(n)]^{2}}{2 \operatorname{var}\left(\widehat{S}_{n} \mid\left(\Gamma_{k}\right)\right)}\right]\right\} .
$$

Proceeding as in the proof of Lemma 6.3 and choosing $\theta$ small enough, the last expression can be bounded by $\mathrm{Cn}^{-r}$ for any $r>0$. This concludes the proof.

The following statement is now a straightforward conclusion from the previous lemma. 
LEMMA 6.6. For every $\gamma>0$ and $r>0$, there are positive constants $\theta$ and $C$ such that, for all $u>0$ and $m=m(u)$,

$$
R_{m}=P\left(\phi\left(\mathbf{Z}_{1}^{(m)}-\gamma \mu\right)>0, A_{m \infty}\right) \leq C m^{-r} .
$$

Moreover,

$$
\lim _{u \rightarrow \infty} \frac{P\left(\phi\left(\mathbf{Z}_{1}^{(m)}-\varepsilon \boldsymbol{\mu}\right)>\varepsilon u\right)}{\psi(u)}=0 .
$$

PROOF. Using properties (2.8) and (4.10) of $\phi$, we obtain the bounds

$$
\begin{aligned}
R_{m} & \leq P\left(\bigcup_{n=m+1}^{\infty}\left\{S_{n}-S_{m}>C \gamma \mu\left(\Gamma_{n}\right)\right\} \cap A_{m n}\right) \\
& \leq \sum_{n=m+1}^{\infty} P\left(S_{n}-S_{m}>C \gamma \mu\left(\Gamma_{n}\right), A_{m n}\right) .
\end{aligned}
$$

Now apply Lemma 6.5 to get (6.11). For $u$ large enough, (6.8) holds for all $j$. Choosing $\theta$ small enough (relative to $\varepsilon$ ), (6.12) follows from (6.11) and Lemma 6.1.

7. More general conditions. In an extended version of this paper we proved Theorem 3.1 under more general conditions on $\rho$ and $\boldsymbol{\mu}$, and the same assumptions on the subadditive functional $\phi$ and the function $T(s, u)$. We cite these conditions here for the sake of completeness and to give an impression how far one can deviate from the regular variation conditions on $H$ and $\boldsymbol{\mu}$. Proofs are given in Braverman, Mikosch and Samorodnitsky (2000). Although it seems possible to extend our results to the more general class of Lévy processes with subexponential tails $H$ (this is known for particular choices of the subadditive functional $\phi$; see Remark 3.4), our methods of proof do not easily extend to the class of subexponential distribution $\mathrm{s}$ with semiexponential tails and for the large class of subadditive functionals studied in Theorem 3.1.

7.1. Assumptions on the Lévy measure $\rho$. We make the following assumptions:

1. Dominance of the right tail of the Lévy measure-We assume that the right tail of the one-dimensional Lévy measure $\rho$ dominates its left tail in the sense that there is a constant $A_{1}>0$ such that

$$
\rho((-\infty,-t]) \leq A_{1} \rho([t, \infty)) \text { for all } t \geq 1 .
$$

2. $\Delta_{2}$ condition-There is an $a_{1}>0$ such that

$$
H(2 u) \geq a_{1} H(u) \text { for all } u \geq 1 .
$$

Notice that the $\Delta_{2}$ condition on $H$ yields a bound from below; it excludes exponential decay of $H(u)$. 
3. Bound from above-There is a $\beta_{1}>0$ such that

$$
H(u)=o\left(u^{-\beta_{1}}\right), \quad u \rightarrow \infty .
$$

7.2. Assumptions on the drift $\boldsymbol{\mu}$. Let $\boldsymbol{\mu}=\{\mu(t), t \geq 0\}$ be a nonnegative function satisfying the following assumptions:

1. Power law bound from below-There are $a_{2}>0$ and $\beta_{2}>\max \left(\beta_{1}^{-1}, 0.5\right)$ such that

$$
\mu(t) \geq a_{2} t^{\beta_{2}}, \quad t>0 .
$$

2. $\Delta_{2}$ condition-There are an $A_{2}>0$ and a $t_{0} \geq 0$ such that

$$
\mu(2 t) \leq A_{2} \mu(t) \quad \text { for all } t \geq t_{0} .
$$

The $\Delta_{2}$ condition on $\mu$ excludes a too rapid (in particular exponential) growth of $\mu$.

3. Quasimonotonicity of $\boldsymbol{\mu}$-There are an $a_{3} \in(0,1]$ and a $t_{0} \geq 0$ such that

$$
\inf _{s \geq t} \mu(s) \geq a_{3} \mu(t) \quad \text { for all } t \geq t_{0} .
$$

\section{REFERENCES}

Bertoin, J. (1996). Lévy Processes. Cambridge Univ. Press.

Bingham N. H., Goldie, C. M. and Teugels, J. L. (1987). Regular Variation. Cambridge Univ. Press.

Braverman, M., Mikosch, T. and Samorodnitsky, G. (2000). Tail probabilities of subadditive functionals acting on Lévy processes. Technical report, Laboratory of Actuarial Mathematics, Univ. Copenhagen. Available as levy.ps.gz under www.math.ku.dk/ $\sim$ mikosch/Preprint/Levy.

Embrechts, P., Goldie, C. and Veraverbeke, N. (1979). Subexponentiality and infinite divisibility. Z. Wahrsch. Verw. Gebiete 49 335-347.

Embrechts, P., Klüppelberg, C. and Mikosch, T. (1997). Modelling Extremal Events for Insurance and Finance. Springer, Berlin.

Embrechts, P. and VeraVERbeKe, N. (1982). Estimates for the probability of ruin with special emphasis on the possibility of large claims. Insurance Math. Econom. $155-72$.

Hüsler, J. and Piterbarg, V. (1999). Extremes of a certain class of Gaussian processes. Stochastic Process. Appl. 83 257-271.

Maruyama, G. (1970). Infinitely divisible processes. Theory Probab. Appl. 15 1-22.

Mikosch, T. and SAmorodnitsky, G. (2000). Ruin probabilities for a random walk with stable stationary ergodic increments. Ann. Probab. 28 1814-1851.

Petrov, V. (1995). Limit Theorems of Probability Theory. Oxford Univ. Press.

Prabhu, N. U. (1998). Stochastic Storage Processes. Queues, Insurance Risk, Dams, and Data Communication, 2nd ed. Springer, New York.

Prokhorov, Y. (1959). An extremal problem in probability theory. Theory Probab. Appl. 4 201204.

Resnick, S. I. (1987). Extreme Values, Regular Variation, and Point Processes. Springer, New York. 
Rosiński, J. and SAMORODNITSKY, G. (1993). Distributions of subadditive functionals of sample paths of infinitely divisible processes. Ann. Probab. 21 996-1014.

S ATO, K. (1999). Lévy Processes and Infinitely Divisible Distributions. Cambridge Univ. Press.

Stout, W. F. (1974). Almost Sure Convergence. Academic Press, New York.

M. BRAVERMAN

DePartment OF MATHEMATics

BEN-GURION UNIVERSITY OF THE NEGEV

P.O. BOX 653

BEER-SHEVA 84105

ISRAEL

E-MAIL:braver@cs.bgu.ac.il
T. MIKOSCH

LABORATORY OF ACTUARIAL MATHEMATICS

UNIVERSITY OF COPENHAGEN

UNIVERSITETSPARKEN 5

DK-2100 COPENHAGEN

DENMARK

E-MAIL: mikosch@math.ku.dk

G. SAMORODNITSKY

SCHOOL OF OPERATIONS RESEARCH AND INDUSTRIAL ENGINEERING

and Department of Statistical Science

CORNELL UNIVERSITY

ITHACA, NEW YORK 14853

E-MAIL: gennady@orie.cornell.edu 